\title{
Multisource SWIPT-based coded cooperation:rate compatible codes and codeword splitting protocol
}

\author{
Shunwai Zhang ${ }^{1 *}$, Lingjun Kong ${ }^{1}$ and Jun Li $^{2}$
}

\begin{abstract}
To achieve a high reliable and energy-saving green communication, we investigate a multisource simultaneous wireless information and power transfer (SWIPT)-based coded cooperation where the relay can realize information decoding and energy harvesting. Firstly, a class of naturally rate compatible low-density parity-check (LDPC) codesquasi-cyclic repeat-accumulate (QC-RA) codes is introduced, and the joint parity-check matrix corresponding to the QC-RA codes employed by the multiple sources and relay is deduced. Based on the joint parity-check matrix, we jointly design the QC-RA codes to cancel all the short girth cycles. Then, by exploiting the rate compatible characteristic of QC-RA codes, we propose a new SWIPT protocol—codeword splitting protocol for the proposed system, which has the characteristics of lower complexity, higher efficiency, no strictly bit synchronization limitation, and less hardware requirement. The results show that the bit error rate (BER) performance of the proposed system employing jointly designed QC-RA codes clearly outperforms that of general RA codes. Theoretical analysis and numerical simulations also demonstrate the superiority of the proposed codeword splitting protocol.
\end{abstract}

Keywords: Coded cooperation, Codeword splitting protocol, LDPC codes, QC-RA codes, SWIPT

\section{Introduction}

Currently, the area of information and communication technology (ICT) plays an important role in the global energy consumption and the greenhouse gas emission [1-3]. In next-generation wireless communication, an unprecedented number of devices will be served and huge amounts of application will be provided, which would result in much more serious challenges. To achieve green communications, it is extremely urgent to explore energy-saving technologies and energy efficiency protocols for next-generation wireless communication. Recently, simultaneous wireless information and power transfer (SWIPT) technology [4-6] which can realize information decoding (ID) and energy harvesting (EH) simultaneously have drawn great attention. In SWIPTbased communication systems, the nodes can harvest the energy from radio frequency (RF) signals for transmission. It is energy-saving and reduces the carbon

\footnotetext{
* Correspondence: swzhang@njupt.edu.cn

${ }^{1}$ College of Telecommunications and Information Engineering, Nanjing University of Posts and Telecommunications, Nanjing 210003, China Full list of author information is available at the end of the article
}

footprint. Hence, SWIPT technology is a new approach to the green communications. Furthermore, the lifetime of nodes with SWIPT technology is not restricted by the grid energy or battery, and they can be placed in the hard-to-reach areas to reduce the communication blind zones and enlarge the coverage.

Researchers have investigated various SWIPT-based communication systems such as cognitive radio network [7], multiple-input multiple-output (MIMO) system [8], and cooperative communication [9]. To exploit the spatial diversity, cooperative communication forms a virtual MIMO by sharing the antennas of different nodes. Hence, SWIPT-based cooperative communication can not only overcome the power limitation but also achieve spatial diversity.

The three main protocols for cooperative communication are the amplify-and-forward (AF) [10], decode-andforward (DF) [11], and coded cooperation [12]. The AF protocol has the advantage of lower implementation complexity. The DF protocol performs better than the AF protocol when the source-relay channel is good enough. Coded cooperation protocol combines channel 
coding and cooperative technology. It obtains both coding gain and spatial diversity gain and achieves the best reliable performance.

References [13-15] investigated SWIPT-based AF cooperative communications. In [13], an AF cooperative communication was considered, where the relay uses the energy harvested from RF signals to assist the source in transmitting information. Furthermore, the power splitting protocol and time switching protocol were proposed to implement SWIPT. For an AF-MIMO cooperative communication in [14], the source and relay adopted orthogonal space-time block codes and designed the joint optimal pre-coders to achieve trade-offs between information decoding and energy harvesting. Based on directional modulation, secure SWIPT-based AF relay network was investigated in [15]. To maximize the secrecy rate, the authors built a twin-level optimization problem and then solved it using a one-dimensional search and semi-definite relaxation. References [16-18] studied SWIPT-based DF cooperative communications. Multi-antenna relay-assisted SWIPT for two-hop DF cooperative transmission was considered in [16]. To maximize the achievable rate, the authors formulated a joint problem of power allocation and power splitting at the multi-antenna relay. Reference [17] investigated SWIPT for a DF full-duplex relay network and studied two models for the battery, i.e., the virtual harvest-usestore model and harvest-use model. Reference [18] formulated a distributed precoding problem for SWIPTbased DF MIMO relay networks. A non-cooperative game was established when only local channel state information (CSI) was required, and the existence and uniqueness of the pure strategy Nash equilibrium solution were proved.

The existing works about SWIPT-based cooperative communication mostly focus on the AF protocol or DF protocol. Generally, compared with AF protocol or DF protocol, coded cooperation protocol is much more suitable when extremely reliable communication is required. Hence, SWIPT-based coded cooperation is an effective way to realize extremely reliable and green communication. However, to the best of our knowledge, the references about SWIPT-based coded cooperation are relatively scarce. Low-density parity-check (LDPC) code $[19,20]$ is adopted as channel coding for the data channel in 5G standard [21]. Repeat-accumulate (RA) code $[22,23]$, as a special class of LDPC codes, not only has the merits of high coding gain, low memory consumption, simple encoding/decoding, and low energy consumption but also possess naturally rate compatible characteristic, which is very suitable for the multisource SWIPT-based coded cooperation. Reference [24] investigated the energy harvesting-based RA-coded cooperative MIMO, where single source and relay are just considered. Hence, the application scenarios are strictly limited. Furthermore, in [24], the relay harvests energy by SWIPT technology via the antenna switching proto$\mathrm{col}$, and it does not apply in the single antenna case. We will consider the multisource SWIPT-based RA coded cooperation and propose a new efficient and lower complexity SWIPT protocol which applies both in the single antenna and multiple antenna cases.

\section{Methods}

In this paper, to achieve a high reliable and energysaving green communication, we focus on the multisource SWIPT-based coded cooperation. To improve the reliability, quasi-cyclic RA (QC-RA) codes are introduced to the system, and the QC-RA codes employed by the sources and relay are jointly designed to further improve the coding gain. To save the energy and achieve the green target, SWIPT technology is implemented at the relay. A new efficient and lower complexity SWIPT protocol is further proposed and investigated. The main contributions are summarized as follows:

(1) We briefly introduce the rate compatible QC-RA codes, and we deduce the joint parity-check matrix corresponding to the QC-RA codes employed by the multiple sources and relay in the multisource SWIPT-based coded cooperation.

(2) Based on the joint parity-check matrix, we jointly design the QC-RA codes to cancel all the short girth cycles. We decompose the joint parity-check matrix into two parts, i.e., QC part and quasi-diagonal (QD) part, and then we propose the algorithm and theorems to design the QC part to cancel all the girth-4 cycles in the joint parity-check matrix absolutely.

(3) By exploiting the rate compatible characteristic of QC-RA codes, we propose a new SWIPT protocol-codeword splitting protocol, which is only operated one time during the whole codeword period. It has the characteristics of lower complexity, higher efficiency, no strictly bit synchronization limitation, and less hardware requirement.

The rest of this paper is organized as follows. In Section 2.1, the general fundamental principle of multisource SWIPT-based QC-RA coded cooperation is presented. Section 2.2 mainly deals with joint design of QC-RA codes for the proposed system. Section 2.3 describes the new SWIPT protocol-codeword splitting protocol. Simulation results and discussion are given in Section 3. Finally, Section 4 concludes the whole paper.

\subsection{System description}

For simplicity, the two-source SWIPT-based coded cooperation is considered in Fig. 1, which can be extended 


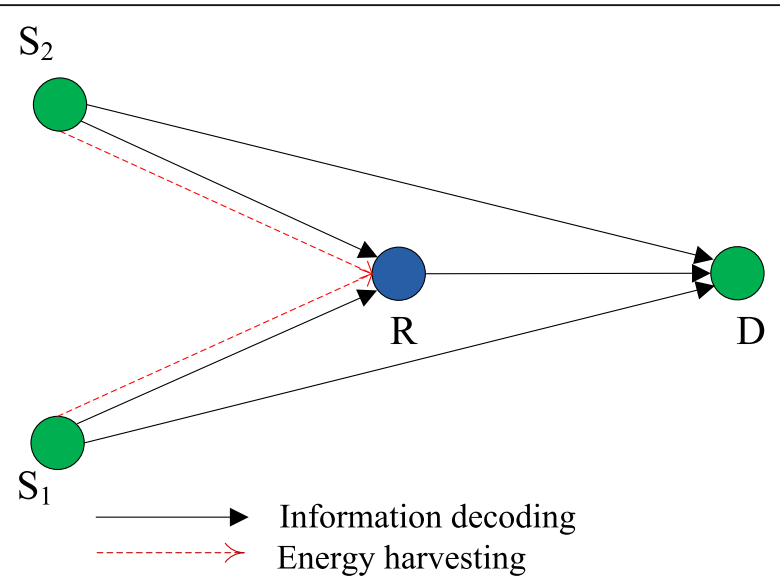

Fig. 1 Two-source SWIPT-based coded cooperation. Two sources $\left(S_{1}\right.$, $\mathrm{S}_{2}$ ) and the destination (D) are powered by external power supply such as the grid. However, the relay (R) cannot access the external power supply and it harvests the energy from the RF signals from two sources via SWIPT technology

easily to multisource multirelay scenarios. Two sources $\left(\mathrm{S}_{1}, \mathrm{~S}_{2}\right)$ and the destination (D) are powered by external power supply such as the grid. However, the relay (R) cannot access the external power supply, and it harvests the energy from the RF signals from two sources via SWIPT technology. In time slot 1 , at the source $S_{1}$, a codeword $c_{1}=\left[\begin{array}{ll}s_{1} & p_{1}\end{array}\right]$ of the first QC-RA encoder (QCRA-1) is sent simultaneously to $\mathrm{R}$ and $\mathrm{D}$ over the broadcast channel. Similarly, in time slot 2 , at the source $\mathrm{S}_{2}$, a codeword $\boldsymbol{c}_{2}=\left[\begin{array}{ll}\boldsymbol{s}_{2} & \boldsymbol{p}_{2}\end{array}\right]$ of the second QC-RA encoder (QC-RA-2) is sent simultaneously to R and D. $\boldsymbol{s}_{i}$ and $\boldsymbol{p}_{i}$ $(i=1,2)$ are the information bits and check bits of $c_{i}$, respectively. In time slot 3 , firstly, the relay $\mathrm{R}$ decodes the information and harvests energy from the two incoming signals of $S_{1}$ and $S_{2}$ via SWIPT technology. Traditionally, the relay combines the decoded messages by network coding [25], such as bitwise exclusive OR (XOR) operation in the Galois field $\mathrm{GF}(2)$. However, this operation will inevitably introduce girth-4 cycles in the joint parity-check matrix. Hence, in the proposed system, the relay $\mathrm{R}$ cascades the information bits $\boldsymbol{s}_{1}$ and $\boldsymbol{s}_{2}$ rather than combines them by network coding and then encodes the cascaded message by another QC-RA encoder (QC-RA-R). Because the information bits $s_{1}$ and $s_{2}$ have been sent to the destination by $S_{1}$ and $S_{2}$, to improve the transmission efficiency, $\mathrm{R}$ transmits only additional parity-check bits to the destination by utilizing the harvested energy.

\subsection{Joint design of rate compatible codes for the multisource SWIPT-based coded cooperation}

In this section, firstly, we briefly introduce a kind of naturally rate compatible LDPC codes-QC-RA codes. Then, we jointly design the QC-RA codes employed by the multiple sources and relay in the SWIPT-based coded cooperation to further improve the coding gain and accelerate the decoding convergence.

\subsubsection{QC-RA codes-a kind of rate compatible LDPC codes}

Assume the sparse parity-check matrix of a QC-RA code has the form as $H=\left[\begin{array}{ll}A & D\end{array}\right] . \quad \boldsymbol{D}$ is a quasidiagonal matrix in which all elements are zero except the elements of the principal diagonal and the elements immediately below this diagonal. $A$ is a quasicyclic sparse matrix which is constructed based on the base matrix and exponent matrix [26]. We refer to $\boldsymbol{A}$ and $\boldsymbol{D}$ as the quasi-cyclic (QC) part and quasidiagonal (QD) part, respectively. The parity-check matrix is shown as follows:

$$
\boldsymbol{H}=\left[\begin{array}{ll}
\boldsymbol{A} & \boldsymbol{D}
\end{array}\right]=\left[\begin{array}{cccccc}
\boldsymbol{I}\left(p_{1,1}\right) & \boldsymbol{I}\left(p_{1,2}\right) & \cdots & \boldsymbol{I}\left(p_{1, L}\right) & 10 & \cdots \\
\boldsymbol{I}\left(p_{2,1}\right) & \boldsymbol{I}\left(p_{2,2}\right) & \cdots & \boldsymbol{I}\left(p_{2, L}\right) & 11 & 0 \\
\vdots & \vdots & \vdots & \vdots & 0 & 0 \\
\boldsymbol{I}\left(p_{J, 1}\right) & \boldsymbol{I}\left(p_{J, 2}\right) & \cdots & \boldsymbol{I}\left(p_{J, L}\right) & \cdots & 11
\end{array}\right]
$$

where

$$
\boldsymbol{I}\left(p_{j, l}\right)=\left\{\begin{array}{c}
\mathbf{0}_{B \times B} \quad \text { if } \quad p_{j, l}=0 \\
\boldsymbol{I}_{B \times B}^{\left(p_{j, l}\right)} \quad \text { if } \quad 0<p_{j, l} \leq B
\end{array} .\right.
$$

$\boldsymbol{I}_{B \times B}^{\left(p_{j, l}\right)} \quad$ is an identity matrix $\boldsymbol{I}_{B \times B}$ with $p_{j}, \quad$-right-cyclicshift.

Definition 1: Let the base matrix $\mathbf{M}(\boldsymbol{A})$ and exponent matrix $\mathrm{E}(\boldsymbol{A})$ of $\boldsymbol{A}$ in $\boldsymbol{H}$ be defined as follows:

$$
\begin{gathered}
\mathbf{M}(\boldsymbol{A})=\left[\begin{array}{cccc}
b_{1,1} & b_{1,2} & \ldots & b_{1, L} \\
b_{2,1} & b_{2,2} & \ldots & b_{2, L} \\
\vdots & \vdots & \ddots & \vdots \\
b_{J, 1} & b_{J, 2} & \ldots & b_{J, L}
\end{array}\right], \\
\mathbf{E}(\boldsymbol{A})=\left[\begin{array}{cccc}
p_{1,1} & p_{1,2} & \cdots & p_{1, L} \\
p_{2,1} & p_{2,2} & \cdots & p_{2, L} \\
\vdots & \vdots & \ddots & \vdots \\
p_{J, 1} & p_{J, 2} & \cdots & p_{J, L}
\end{array}\right],
\end{gathered}
$$

where

$$
b_{j, l}=\left\{\begin{array}{c}
0 \text { if } p_{j, l}=0 \\
1 \text { if } p_{j, l} \neq 0
\end{array}, \quad 1 \leq j \leq J, 1 \leq l \leq L .\right.
$$

\subsubsection{Joint design of QC-RA codes}

Assume $\boldsymbol{H}_{1}=\left[\begin{array}{ll}A & D_{1}\end{array}\right], \boldsymbol{H}_{2}=\left[\begin{array}{ll}B & D_{2}\end{array}\right]$, and $\boldsymbol{H}_{R}=\left[\begin{array}{lll}C_{1} & C_{2} & D_{3}\end{array}\right]$ are the parity-check matrices corresponding to QC-RA-1, QC-RA-2, and QC-RA-R employed by $S_{1}, S_{2}$, and R, respectively, and their corresponding codewords are $\boldsymbol{c}_{1}=\left[\begin{array}{ll}\boldsymbol{s}_{1} & \boldsymbol{p}_{1}\end{array}\right], \boldsymbol{c}_{2}=\left[\begin{array}{ll}\boldsymbol{s}_{2} & \boldsymbol{p}_{2}\end{array}\right]$, and $\boldsymbol{c}_{R}=\left[\begin{array}{lll}\boldsymbol{s}_{1} \boldsymbol{s}_{2} & \boldsymbol{p}_{R}\end{array}\right]$. From the viewpoint of the SWIPT-based coded cooperation system, 
the overall codeword at the destination is $\boldsymbol{c}=\left[\begin{array}{lll}\boldsymbol{c}_{1} & \boldsymbol{c}_{2} & \boldsymbol{p}_{R}\end{array}\right]$. Define the joint parity-check matrix of $\boldsymbol{c}$ as $\tilde{\boldsymbol{H}}$. We have $\tilde{\boldsymbol{H}} \boldsymbol{c}=0$. According to the following parity-check relationship

$$
\begin{aligned}
& \boldsymbol{H}_{1} \boldsymbol{c}_{1}=\mathbf{0}, \\
& \boldsymbol{H}_{2} \boldsymbol{c}_{2}=\mathbf{0}, \\
& \boldsymbol{H}_{R} \boldsymbol{c}_{R}=\mathbf{0},
\end{aligned}
$$

the joint parity-check matrix $\tilde{\boldsymbol{H}}$ is achieved as:

$$
\tilde{\boldsymbol{H}}=\left[\begin{array}{lllcl}
\boldsymbol{A} & \boldsymbol{D}_{1} & \mathbf{0} & \mathbf{0} & \mathbf{0} \\
\mathbf{0} & \mathbf{0} & \boldsymbol{B} & \boldsymbol{D}_{2} & \mathbf{0} \\
\boldsymbol{C}_{1} & \mathbf{0} & \boldsymbol{C}_{2} & \mathbf{0} & \boldsymbol{D}_{3}
\end{array}\right]
$$

The joint Tanner graph corresponding to the joint parity-check matrix $\tilde{\boldsymbol{H}}$ is illustrated in Fig. 2.

While we analyze the short cycles in the joint Tanner graph corresponding to $\tilde{\boldsymbol{H}}$, exchanging any two columns of $\tilde{\boldsymbol{H}}$ does not influence the status of the cycles. For simplicity, we firstly exchange the second and third columns of $\tilde{\boldsymbol{H}}$ and then decompose $\tilde{\boldsymbol{H}}$ into $\tilde{\boldsymbol{H}}_{\mathrm{Q}} \mathrm{C}$ and $\tilde{\boldsymbol{H}}_{\mathrm{Q}} \mathrm{D}$.

$$
\begin{aligned}
\tilde{\boldsymbol{H}}_{\mathrm{QC}} & =\left[\begin{array}{ll}
\boldsymbol{A} & \mathbf{0} \\
\mathbf{0} & \boldsymbol{B} \\
\boldsymbol{C}_{1} & \boldsymbol{C}_{2}
\end{array}\right], \\
\tilde{\boldsymbol{H}}_{\mathrm{QD}} & =\left[\begin{array}{ccc}
\boldsymbol{D}_{1} & \mathbf{0} & \mathbf{0} \\
\mathbf{0} & \boldsymbol{D}_{2} & \mathbf{0} \\
\mathbf{0} & \mathbf{0} & \boldsymbol{D}_{3}
\end{array}\right] .
\end{aligned}
$$

To further improve the coding gain, we design the joint parity-check matrix $\tilde{\boldsymbol{H}}$ to cancel all the girth- 4 cycles in the joint Tanner graph. Firstly, we jointly design the base matrix of QC part $\mathbf{M}\left(\tilde{\boldsymbol{H}}_{\mathrm{QC}}\right)$ by Algorithm 1 to cancel the girth-4 cycles as much as possible. Secondly,

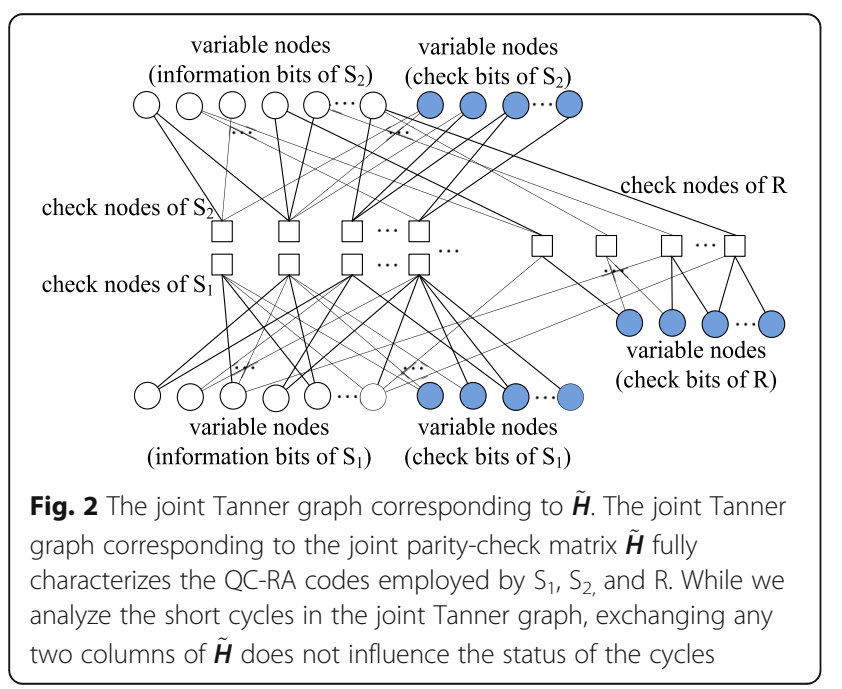

if there are still remaining girth- 4 cycles in $\mathbf{M}\left(\tilde{\boldsymbol{H}}_{\mathrm{QC}}\right)$, we further design the exponent matrix of QC part $\mathbf{E}\left(\tilde{\boldsymbol{H}}_{\mathrm{QC}}\right)$ by Theorem 1 and Theorem 2 to cancel all the girth- 4 cycles absolutely [27].

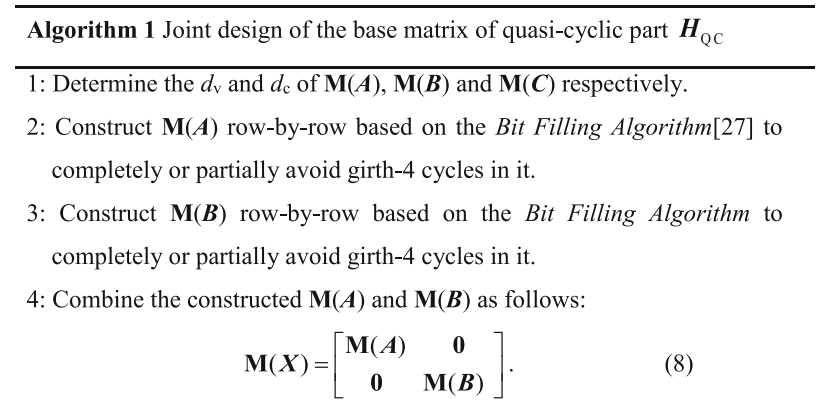

5: Regard $\mathbf{M}(\boldsymbol{X})$ as the rows already filled and $\mathbf{M}(\boldsymbol{C})=\left[\mathbf{M}\left(\boldsymbol{C}_{1}\right)\left(\mathbf{M} \boldsymbol{C}_{2}\right)\right]$ as the rows to be further filled, and then continue to construct $\mathbf{M}(\boldsymbol{C})$ row-by-row based on the Bit Filling Algorithm.

6: According to the constructed $\mathbf{M}(\boldsymbol{A}), \mathbf{M}(\boldsymbol{B})$ and $\mathbf{M}(\boldsymbol{C})$, we achieve

$$
\mathbf{M}\left(\boldsymbol{H}_{\mathrm{QC}}\right)=\left[\begin{array}{cc}
\mathbf{M}(\boldsymbol{A}) & \mathbf{0} \\
\mathbf{0} & \mathbf{M}(\boldsymbol{B}) \\
\mathbf{M}\left(\boldsymbol{C}_{1}\right) & \mathbf{M}\left(\boldsymbol{C}_{2}\right)
\end{array}\right] .
$$

7: The base matrix of QC part of the joint parity check matrix $\boldsymbol{H}$ is jointly designed.

Theorem 1: Assume there is a remaining girth-4 cycle in $\mathbf{M}\left(\tilde{\boldsymbol{H}}_{\mathrm{QC}}\right)$, whose corresponding shift values are $p_{j, l}, p_{j+k, l}$, $p_{j, l+t}$, and $p_{j+k, l+t}$. To avoid girth- 4 cycles in $\tilde{\boldsymbol{H}}_{\mathrm{Q}}$ C, a necessary and sufficient condition that they should satisfy is

$$
\left(p_{j, l}-p_{j+k, l}\right)+\left(p_{j, l+t}-p_{j+k, l+t}\right) \neq 0 \bmod B .
$$

The proof is referred in [26].

Theorem 2: Assume there are two upper and lower adjacent "1"s in $\mathbf{M}\left(\tilde{\boldsymbol{H}}_{\mathrm{QC}}\right)$, whose corresponding shift values are $p_{j, l}, p_{j+1, l}$. To cancel girth-4 cycles between $\tilde{\boldsymbol{H}}_{\mathrm{Q} ~ \mathrm{C}}$ and $\tilde{\boldsymbol{H}}_{\mathrm{Q}}$, a necessary and sufficient should be satisfied is

$$
p_{j, l}-p_{j+1, l} \neq 1 \bmod B .
$$

The proof in detail can be referred in [24].

\subsection{Codeword splitting protocol for the multisource SWIPT-based coded cooperation}

In the proposed system, assume two sources employ the jointly designed QC-RA codes with the same code length $N=K+M$ and code rate $r=K / N=K /(K+M)$, and the codeword $\boldsymbol{c}=\left(s_{1}, s_{2}, \cdots, s_{K}, p_{1}, p_{2}, \cdots, p_{M}\right)$ is shown in 


\begin{tabular}{l}
\hline$\left(s_{1}, s_{2}, \cdots, s_{K}\right)$ \\
information bits $\left(p_{1}, p_{2}, \cdots, p_{m}\right)$
\end{tabular}

Fig. 3. We explore the rate compatible characteristic of QC-RA codes. Assume the quality of $S_{1}-R$ channels is improved, it can support the code rate up to $r_{1}=K /(K+m)$. The so called codeword splitting protocol for SWIPT technology is described as follows. The codeword $\boldsymbol{c}=\left(s_{1}\right.$, $\left.s_{2}, \cdots, s_{K}, p_{1}, p_{2}, \cdots, p_{M}\right)$ is split into two parts, i.e., the subcodeword $\hat{\boldsymbol{c}}=\left(s_{1}, s_{2}, \cdots, s_{K}, p_{1}, p_{2}, \cdots, p_{m}\right)$ with code rate $r_{1}$ and the remaining $M_{E H}=M-m$ check bits $\left(p_{m+1}, p_{m+}\right.$ $\left.{ }_{2}, \cdots, p_{M}\right)$. The sub-codeword $\hat{\boldsymbol{c}}$ is only needed for information decoding, and the remaining $M-m$ check bits are used for energy harvesting.

As shown in Fig. 4, for the power splitting protocol, the power of the received signal corresponding to each bit of the codeword is separated into two parts, one for information decoding and the other for energy harvesting. It is difficult to be implemented in hardware, and the energy utilization efficiency is limited. For the time switching protocol, the ID and EH modes have to be switched during each bit transmission period. The implementation complexity is high, and the strictly bit synchronization is required. The antenna switching protocol does not apply in the single antenna case. It can be seen that the power splitting protocol and the time switching protocol are both carried out during each bit period. However, the proposed codeword splitting protocol is only operated one time during the whole codeword period. Hence, the codeword splitting protocol is with the characteristics of lower complexity, higher efficiency, no strictly bit synchronization limitation, and less hardware requirement.

\section{Results and discussion}

We investigate the performance of multisource SWIPTbased coded cooperation by numerical simulations in this section. $S_{1}-R, S_{2}-R, S_{1}-D, S_{2}-D$, and $R-D$ are all

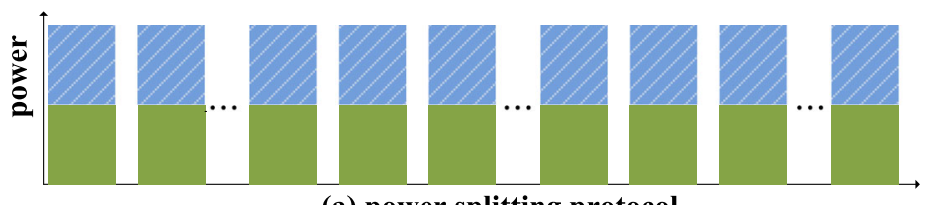

(a) power splitting protocol time
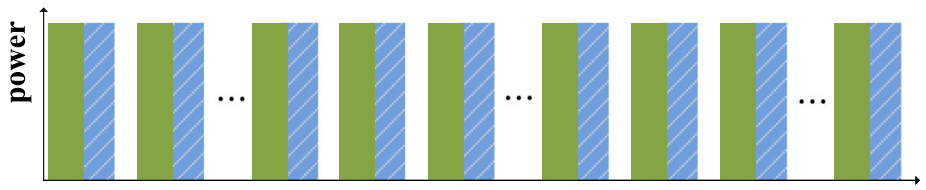

(b) time switching protocol

time

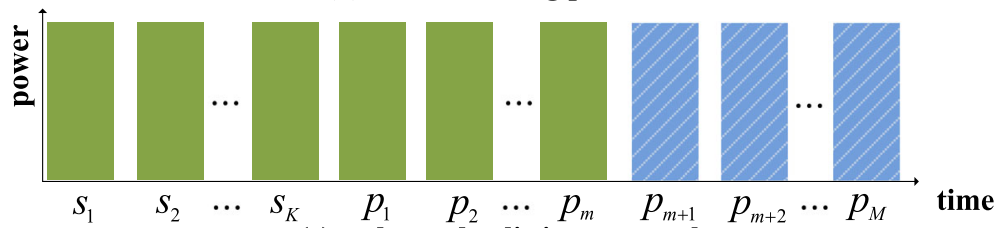

(c) codeword splitting protocol

Fig. 4 Comparison of the proposed codeword splitting protocol and other SWIPT protocols. It can be seen that the power splitting protocol and the time switching protocol are both carried out during each bit period. However, the proposed codeword splitting protocol is only operated one time during the whole codeword period. Hence, the codeword splitting protocol is with the characteristics of lower complexity, higher efficiency, no strictly bit synchronization limitation, and less hardware requirement 
additive white Gaussian noise (AWGN) channels. The signal to noise ratios (SNRs) of $S_{1}-D$ and $S_{2}$-D are the same. The $S N R$ s of $S_{1}-R$ and $S_{2}-R$ determine how many bits from $S_{1}$ and $S_{2}$ can be used for $E H$ at the relay, and the SNR of R-D depends on the number of bits for $\mathrm{EH}$ at the relay. The joint iterative decoding algorithm [28] and binary phase shift keying (BPSK) modulation are assumed at the destination. For two-source SWIPT-based coded cooperation, QC-RA codes at the sources and relay are given in Table 1.

\subsection{BER comparison of SWIPT-based jointly designed QC-} RA coded cooperation and general RA coded cooperation We compare the BER performance of SWIPT-based jointly designed QC-RA coded cooperation and general RA coded cooperation. General RA codes and jointly designed QC-RA codes have the same code length and code rate codes as shown in Table 1 . Their parity-check matrices both have quasi-cyclic structure. The relay uses sub-codeword with 300 bits for ID and the rest 100 check bits for EH. It is shown in Fig. 5 when the number of decoding iterations is one, the BER curves of the jointly designed QC-RA codes and the general RA codes are almost the same. It is because the extrinsic information is not exchanged sufficiently during the iterative decoding, and the influence of short cycles does not appear obviously. Figure 5 also illustrates that the BER performance of the jointly designed QC-RA coded cooperation clearly outperforms that of general RA coded cooperation when the number of decoding iterations is two or ten. This is because in the jointly designed QC-RA codes, all girth-4 cycles are cancelled, and there are neither girth-4 cycles in the single QC-RA codes nor girth4 cycles between them. Hence, when the joint iterative decoding is implemented at the destination, higher coding gain is achieved, and the gain increases with the number of decoding iterations rising.

\subsection{BER comparison of SWIPT-based QC-RA coded cooperation with codeword splitting protocol and power splitting protocol}

In this part, we compare the proposed SWIPT-based QC-RA coded cooperation with codeword splitting protocol and the power splitting protocol in [17]. The relay uses sub-codeword with 300 bits for ID and the rest 100 check bits for $\mathrm{EH}$. The number of decoding iterations is ten. For a fair comparison, in the power splitting protocol, we assume $1 / 4$ power of each bits of the

Table 1 QC-RA codes at the sources and relay

QC-RA codes for the SWIPT-based coded cooperation

$\boldsymbol{H}_{1}\left(\right.$ or $\left.\boldsymbol{H}_{2}\right)=\left[\begin{array}{ll}\boldsymbol{A}_{200 \times 200} & \left.\boldsymbol{D}_{200 \times 200}\right], r_{S}=1 / 2, N=400, B=50\end{array}\right.$

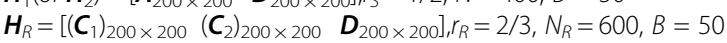

total codeword is used to harvest energy. When the energy utilization ratio is $\eta=1$, it is shown in Fig. 6 that the BER performance of the codeword splitting protocol is almost the same with the power splitting protocol. For the power splitting protocol, as described in Section 2.3, firstly, the relay has to split the power bit by bit for ID and $\mathrm{EH}$ and then collects the EH energy from all bits for transmission. Hence, compared with the codeword splitting protocol, the energy utilization ratio of the power splitting protocol actually decreases. Furthermore, the time delay at the relay is longer, and the complexity is higher. When the energy utilization ratio of the power splitting protocol decreases to 0.9 or 0.6 , the BER performance of codeword splitting protocol is much superior to that of the power splitting protocol.

\subsection{BER performance of SWIPT-based QC-RA coded cooperation exploiting rate compatible characteristic} For the codeword splitting protocol, at the relay, the number of check bits for $\mathrm{EH}$ adapts to the quality of $\mathrm{S}_{i^{-}}$ $R$ channels. We assume the quality of $S_{1}-R$ channel is fixed and it can support the code rate up to $r_{1}=2 / 3$. The quality of $\mathrm{S}_{2}-\mathrm{R}$ channel varies and it adapts to support the code rate $r_{2}=2 / 3,4 / 5,1$. By the rate compatible characteristic of QC-RA codes, $M_{E H}=M-m=100$, 150, 200 check bits can be exploited for $E H$, respectively. In Fig. 7, it is shown that the BER of the proposed system decreases sharply with the quality of $\mathrm{S}_{2}-\mathrm{R}$ channel becomes higher from supporting code rate $r_{2}=2 / 3$ to supporting code rate $r_{2}=1$. This is because the more check bits exploited for $\mathrm{EH}$ by the rate compatible characteristic of QC-RA codes, the more energy harvested at the relay.

\subsection{BER comparison of SWIPT-based coded cooperation and point to point system over AWGN channels or Rayleigh fading channels}

In this part, we compare the BER performance of SWIPT-based coded cooperation and point-to-point (noncooperation) system over AWGN channels or Rayleigh fading channels, which are block fading with perfect channel state information at the destination. The fading coefficient for each channel remains constant over each codeword. SWIPT-based coded cooperation employs the jointly designed QC-RA codes as shown in Table 1, and the relay uses sub-codeword with 300 bits for ID and the rest 100 check bits for EH. The point-to-point system without SWIPT-based relay employs single QC-RA code whose girth-4 cycles are also cancelled. The number of decoding iterations is ten.

In Fig. 8, it is shown that the BER performance of SWIPT-based coded cooperation clearly outperforms 


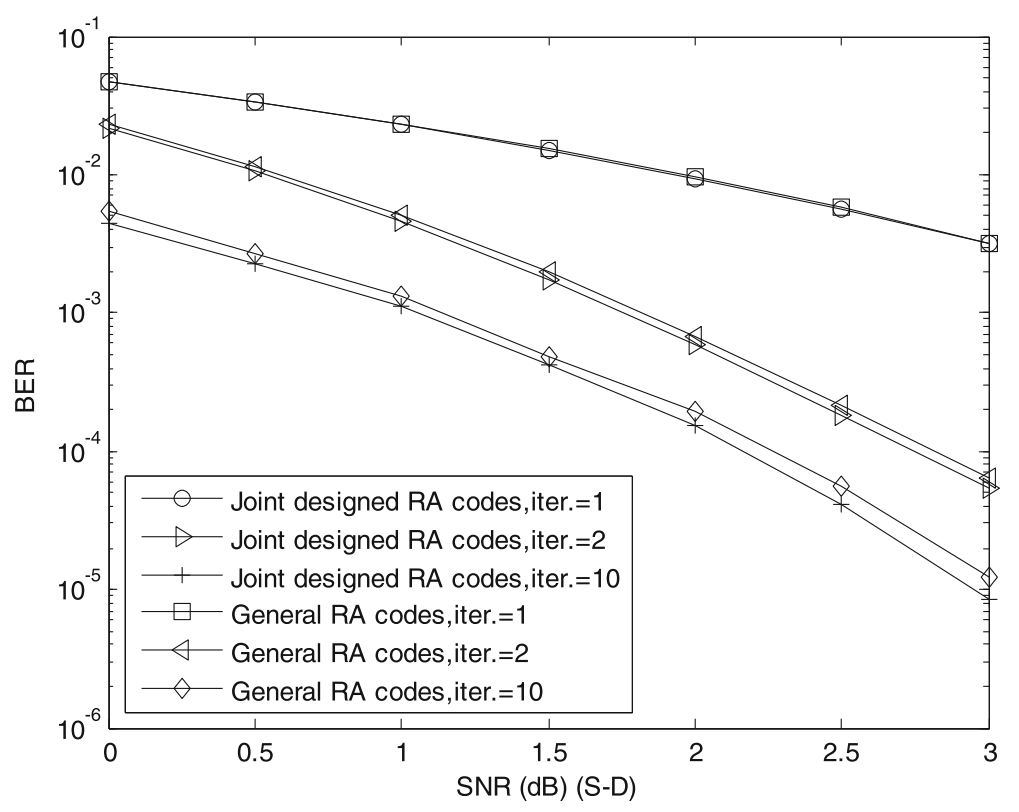

Fig. 5 BER comparison of SWIPT-based jointly designed QC-RA coded cooperation and general RA coded cooperation. It illustrates that the BER performance of the jointly designed QC-RA coded cooperation clearly outperforms that of general RA coded cooperation when the number of decoding iterations is two or ten

that of the point-to-point system over AWGN channels. For example, at the SNR $=3 \mathrm{~dB}$, compared with the point-to-point system, the BER of the coded cooperation drops from about $2 \times 10^{-5}$ to $8 \times 10^{-6}$. It demonstrates the superiority of the investigated scheme over AWGN channels. We also compare them over Rayleigh fading channels. It is demonstrated that the BER performance of coded cooperation is much better than that of the point-to-point system. For example, at the BER $=10^{-3}$, it achieves about $2 \mathrm{~dB}$ gain. What is more, the results also

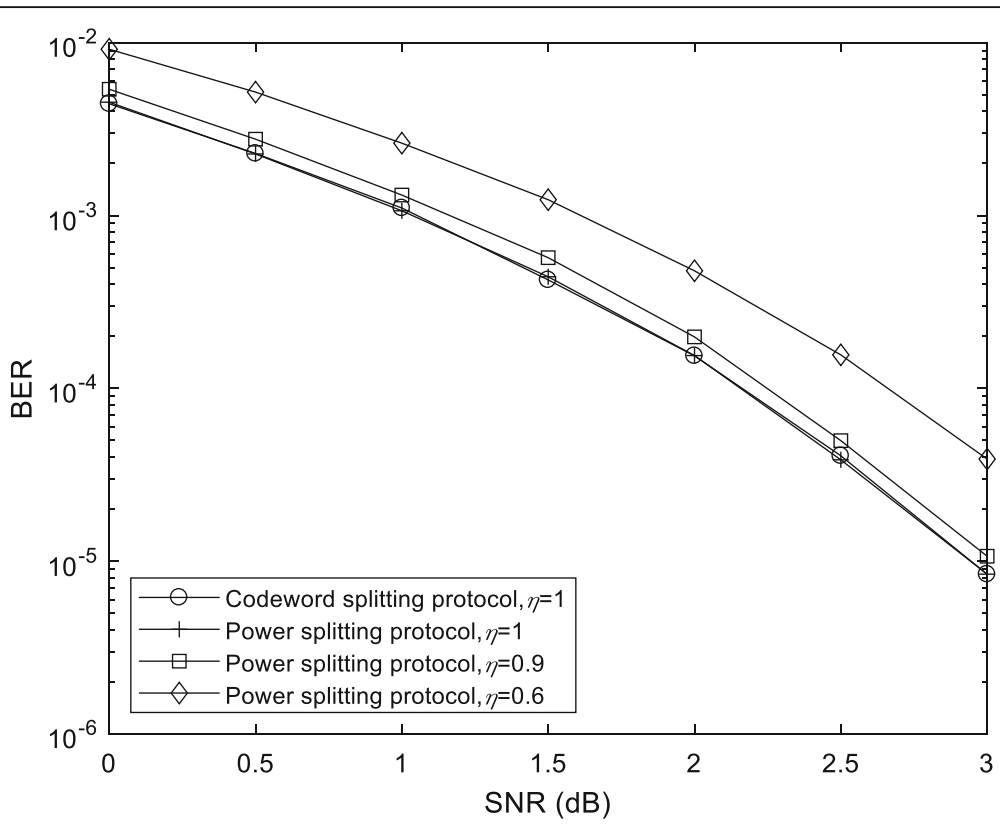

Fig. 6 BER comparison of SWIPT-based QC-RA coded cooperation with codeword splitting protocol and power splitting protocol. When the energy utilization ratios are the same, it is shown that the BER performance of the codeword splitting protocol is almost the same with the power splitting protocol. However, the energy utilization ratio of the power splitting protocol actually decreases, and the time delay at the relay is longer and the complexity is higher 


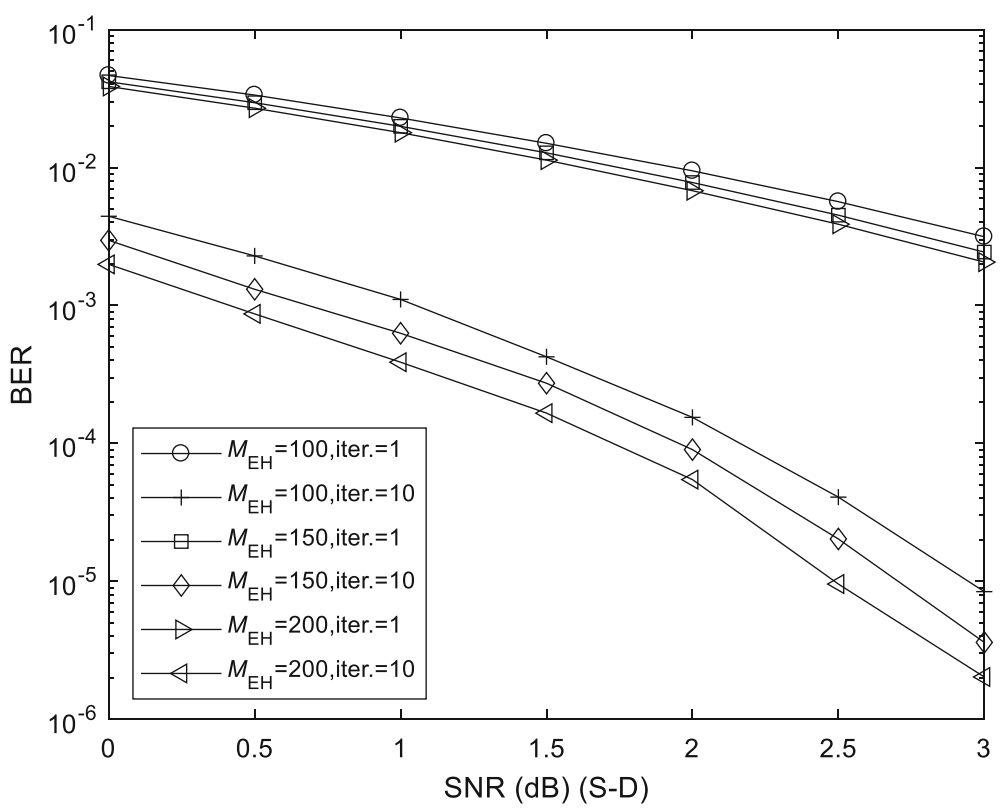

Fig. 7 BER performance of SWIPT-based QC-RA coded cooperation exploiting rate compatible characteristic. It is shown that the BER of the proposed system decreases sharply with the quality of $S_{2}-R$ channel becomes higher from supporting code rate $r_{2}=2 / 3$ to supporting code rate $r_{2}=1$. The more check bits exploited for EH by the rate compatible characteristic of QC-RA codes, the more energy harvested at the relay

show that the SWIPT-based coded cooperation achieves a higher diversity gain.

\section{Conclusion}

In this paper, we have investigated the multisource SWIPT-based QC-RA coded cooperation. It combines the SWIPT, advanced channel coding, and cooperation technologies. Hence, the investigated system can achieve high coding gain, spatial diversity gain, and high energy efficiency. It is a new approach to the high reliable and energy-saving green characteristics of the next generation wireless communications. We deduced the joint

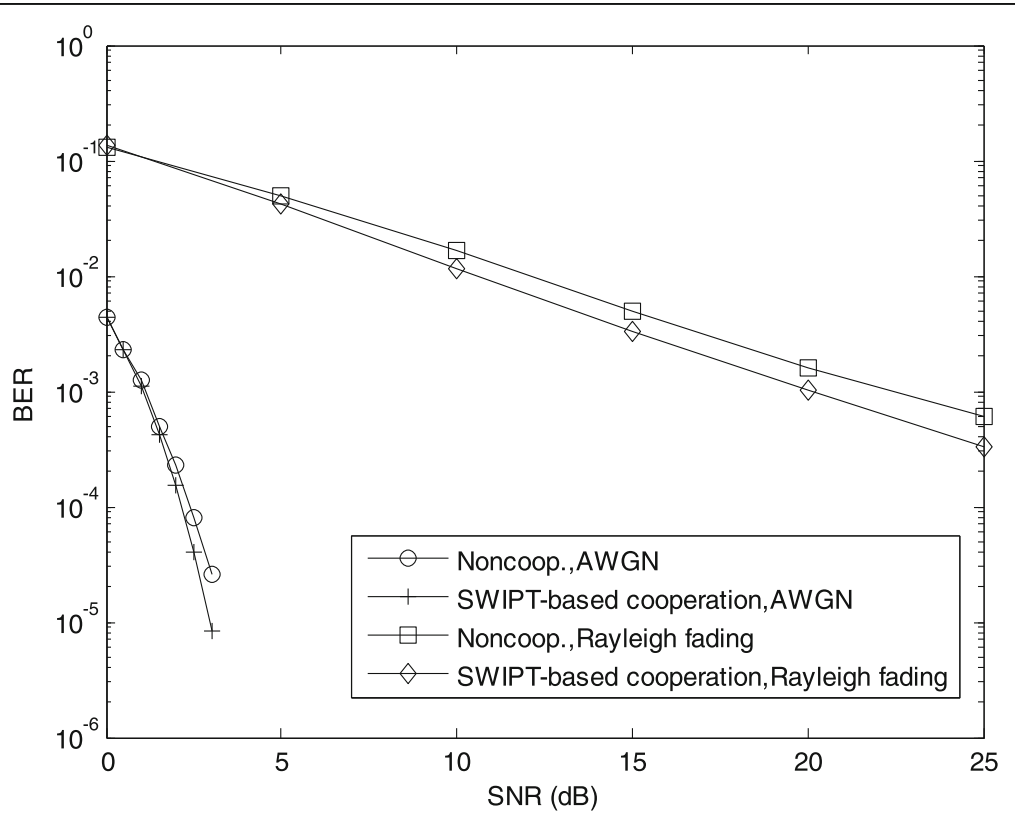

Fig. 8 BER Comparison of SWIPT-based coded cooperation and point-to-point system over AWGN channels or Rayleigh fading channels. It is shown that the BER performance of SWIPT-based coded cooperation clearly outperforms that of the point-to-point system over AWGN channels or Rayleigh fading channels. Furthermore, the SWIPT-based coded cooperation achieves a higher diversity gain over Rayleigh fading channels 
parity-check matrix corresponding to the QC-RA codes employed by the multiple sources and relay, based on which we jointly designed the rate compatible QC-RA codes to cancel all the girth-4 cycles. Furthermore, by exploring the rate compatible characteristic of QC-RA codes, we proposed a new SWIPT protocol-codeword splitting protocol for the proposed system. Theoretical analysis and numerical simulations demonstrated the superiority of the designed QC-RA codes and the proposed codeword splitting protocol.

\section{Abbreviations}

AF: Amplify-and-forward; AWGN: Additive white Gaussian noise; BER: Bit error rate; BPSK: Binary phase shift keying; DF: Decode-and-forward; EH: Energy harvesting; ICT: Information and communication technology; ID: Information decoding; LDPC: Low-density parity-check; MIMO: Multiple-input multipleoutput; QC-RA: Quasi-cyclic repeat-accumulate; SWIPT: Simultaneous wireless information and power transfer

\section{Acknowledgements}

The authors wish to thank the editor and the anonymous reviewers for their valuable suggestions on improving this paper.

\section{Authors' contributions}

Shunwai Zhang is the main writer of this paper and proposed the main idea. Lingjun Kong and Jun Li revised and checked the whole manuscript. All authors read and approved the final manuscript.

\section{Authors' information}

Shunwai Zhang received the B.E. degree in Electronic Engineering from Nanjing University of Technology, Nanjing, China, in 2008, and the Ph.D. degree in Telecommunications from Nanjing University of Aeronautics and Astronautics, Nanjing, China, in 2014. He joined Nanjing University of Posts and Telecommunications as an Assistant Professor since 2014, and then as an Associate Professor since 2017. From 2016 to 2017, he was a research scientist with the Department of Electrical Engineering, University of Texas at Dallas, USA. He has authored or co-authored over 20 research papers. His research interest includes advanced channel coding, cooperative communications, and green communications.

Lingjun Kong received the M.S. and the Ph.D. degree in Electrical Engineering from Beijing Jiaotong University, Beijing, China, in 2007 and 2011, respectively. He had been a research fellow at Nanyang Technical University from Feb. 2012 to Nov. 2013, and a visiting scholar in the Center for Memory Recording Research (CMRR) at University of California, San Diego, USA, from Dec. 2016 to Dec. 2017. He is currently an associate professor with College of Telecommunication and Information Engineering, Nanjing University of Posts and Telecommunications, Nanjing, China. His current research interests include coding theory, communication theory, and signal processing for various data storage and wireless communication systems

Jun Li received Ph.D. degree in Electronic Engineering from Shanghai Jiao Tong University, Shanghai, P. R. China, in 2009. From January 2009 to June 2009, he worked in the Department of Research and Innovation, Alcatel Lucent Shanghai Bell as a research scientist. From June 2009 to April 2012, he was a postdoctoral fellow at the School of Electrical Engineering and Telecommunications, the University of New South Wales, Australia. From April 2012 to June 2015, he is a research fellow at the School of Electrical Engineering, the University of Sydney, Australia. From June 2015 to now, he is a professor at the School of Electronic and Optical Engineering, Nanjing University of Science and Technology, Nanjing, China. His research interests include network information theory, channel coding theory, wireless network coding, and cooperative communications.

\section{Funding}

This work was supported in part by the National Natural Science Foundation of China $(61501256,61501250)$, the Natural Science Foundation of Jiangsu Province (BK20150857), the NUPTSF (NY219073), and the China Scholarship Council (201608320093).

\section{Availability of data and materials}

The authors declare that all the data and materials in this manuscript are available from the author.

\section{Competing interests}

The authors declare that they have no competing interests.

\section{Author details}

${ }^{1}$ College of Telecommunications and Information Engineering, Nanjing University of Posts and Telecommunications, Nanjing 210003, China. ${ }^{2}$ School of Electronic and Optical Engineering, Nanjing University of Science and Technology, Nanjing 210094, China.

Received: 21 October 2019 Accepted: 13 February 2020 Published online: 04 March 2020

\section{References}

1. S. Zhang, Q. Wu, S. Xu, et al., Fundamental green tradeoffs: progresses, challenges, and impacts on $5 \mathrm{G}$ networks. IEEE Commun Surv Tut 19(1), 3356 (2017)

2. K. Singh, A. Gupta, T. Ratnarajah, et al., A general approach toward green resource allocation in relay-assisted multiuser communication networks. IEEE Trans Wireless Commun 17(2), 848-862 (2018)

3. P. Gandotra, R. Jha, S. Jain, Green communication in next generation cellular networks: a survey. IEEE Access 5, 11727-11758 (2017)

4. T.D.P. Perera, D.N.K. Jayakody, S.K. Sharma, et al., Simultaneous wireless information and power transfer (SWIPT): recent advances and future challenges. IEEE Commun. Surv. Tut. 20(1), 264-302 (2018)

5. Z. Ali, G.A.S. Sidhu, S. Zhang, et al., Achieving green transmission with energy harvesting based cooperative communication. IEEE Access 6, 2750727517 (2018)

6. L. Shi, Y. Ye, R.Q. Hu, et al., Energy efficiency maximization for SWIPT enabled two-way DF relaying. IEEE Signal Process Lett 26(5), 755-759 (2019)

7. S. Park, H. Kim, D. Hong, Cognitive radio networks with energy harvesting IEEE Trans Wireless Commun 12(3), 1386-1397 (2013)

8. G. Dong, H. Zhang, D. Yuan, Downlink achievable rate of massive MIMO enabled SWIPT systems over Rician channels. IEEE Commun Lett 22(3), 578581 (2018)

9. T. Li, P. Fan, K. Letaief, Outage probability of energy harvesting relay-aided cooperative networks over Rayleigh fading channel. IEEE Trans Veh Technol 65(2), 972-978 (2016)

10. J. N. Laneman, G. W. Wornell, and D. N. C. Tse, An efficient protocol for realizing cooperative diversity in wireless networks. in Proc. IEEE Int. Symp. Inf. Theory, Washing DC, U.S.A., 294-297 (2001)

11. T. M. Cover, and A. El Gamal, Capacity theorems for the relay channel. IEEE Trans. Inf. Theor IT-25( 5), 572-584 (1979)

12. A. Sendonaris, E. Erkip, B. Aazhang, User cooperation diversity - Part I: system description. IEEE Trans Commun 51(11), 1927-1938 (2003)

13. X.Y. Zhou, S. Durrani, et al., Relaying protocols for wireless energy harvesting and information processing. IEEE Trans Wireless Commun 12(7), 3622-3636 (2013)

14. B.K. Chalise, W.K. Ma, Y.M.D. Zhang, et al., Optimum performance boundaries of OSTBC based AF-MIMO relay system with energy harvesting receiver. IEEE Trans Signal Process 61(17), 4199-4213 (2013)

15. X. Zhou, J. Li, F. Shu, et al., Secure SWIPT for directional modulation-aided AF relaying networks. IEEE J Sel Areas Commun 37(2), 253-268 (2019)

16. Y. Liu, Joint resource allocation in SWIPT-based multiantenna decode-andforward relay networks. IEEE Trans Veh Technol 66(10), 9192-9200 (2017)

17. H.W. Liu, KJ. Kim, K.S. Kwak, et al., Power splitting-based SWIPT with decode-and-forward full-duplex relaying. IEEE Trans Wireless Commun 15(11), 7561-7757 (2016)

18. B. Fang, W. Zhong, S. Jin, Z.P. Qian, et al., Game-theoretic precoding for SWIPT in the DF-based MIMO relay networks. IEEE Trans Veh Technol 65(9), 6940-6948 (2016)

19. P. Chen, K. Cai, S. Zheng, Rate-adaptive protograph LDPC codes for multilevel-cell NAND flash memory. IEEE Commun Lett 22(6), 1112-1115 (2018)

20. Y. Fang, P. Chen, G. Cai, et al., Outage-limit-approaching channel coding for future wireless communications: root-protograph low-density parity-check codes. IEEE Veh Technol Mag 14(2), 85-93 (2019)

21. T. Richardson, S. Kudekar, Design of low-density parity check codes for 56 new radio. IEEE Commun Mag 56(3), 28-34 (2018) 
22. S.T. Brink, G. Kramer, Design of repeat-accumulate codes for iterative detection and decoding. IEEE Trans Signal Process 51(11), 2764-2772 (2003)

23. M. Qiu, L. Yang, Y. Xie, et al., On the design of multi-dimensional irregular repeat- accumulate lattice codes. IEEE Trans Commun 66(2), 478-492 (2018)

24. S. Zhang, F. Yang, R. Song, Energy-harvesting-based RA-coded cooperative MIMO: codes design and performance analysis. Digit Signal Process 60, 5662 (2017)

25. P. Chen, Z. Xie, Y. Fang et al., Physical-layer network coding: an efficient technique for wireless communications. IEEE Network, early access, 1-7 (2019)

26. S. Zhang, F. Yang, L. Tang, et al., Joint design of QC-LDPC codes for coded cooperation system with joint iterative decoding. Int J Electron 103(3), 384405 (2016)

27. J. Campello, D. S. Modha, and S.Rajagopalan, Designing LDPC codes using bit-filling. 2001 IEEE Int. Conf. Commun. 55-59 (2001)

28. S. Zhang, F. Yang, L. Tang, Network-coding-based multisource multirelay LDPC-coded cooperative MIMO. Trans Emerg Telecommun Technol 16(3), 491-502 (2015)

\section{Publisher's Note}

Springer Nature remains neutral with regard to jurisdictional claims in published maps and institutional affiliations.

\section{Submit your manuscript to a SpringerOpen ${ }^{\circ}$ journal and benefit from:}

- Convenient online submission

- Rigorous peer review

- Open access: articles freely available online

High visibility within the field

- Retaining the copyright to your article

Submit your next manuscript at $\boldsymbol{\nabla}$ springeropen.com 